
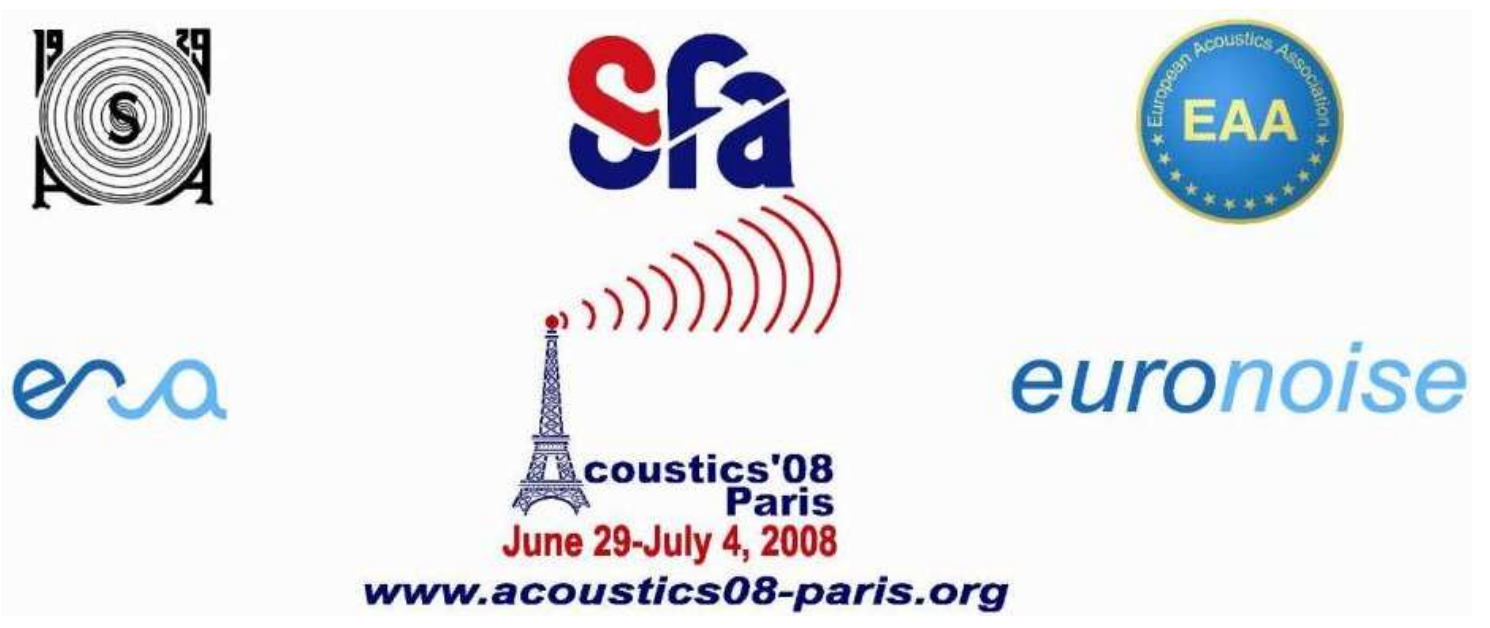

\title{
Sound amplification in a lined duct with flow: PIV measurements
}

D. Marx ${ }^{\mathrm{a}}$, Y. Aurégan ${ }^{\mathrm{b}}$, H. Bailliet ${ }^{\mathrm{a}}$ and J.-C. Valière ${ }^{\mathrm{c}}$

aLaboratoire d'Etudes Aerodynamiques - CNRS, Bat K, 40 avenue du recteur Pineau, 86022 Poitiers, France

bLaboratoire d'Acoustique de l'Université du Maine, Avenue Olivier Messiaen, 72085 Le Mans, France

${ }^{\mathrm{c}}$ Laboratoire d'Etudes Aérodynamiques (LEA), Université de Poitiers, ENSMA, CNRS, Bat K, 40 avenue du recteur Pineau, 86022 Poitiers, France

david.marx@lea.univ-poitiers.fr 
An experimental investigation of the acoustic behavior of a liner in a rectangular channel with grazing flow has been conducted. The liner is a locally reacting structure. When increasing the velocity of the grazing flow the transmission coefficient increases at resonance frequency. The transmission coefficient can become close to 1 and the liner produces acoustical energy instead of dissipating it. This amplification of the sound wave is accompanied by an increase in the stationary pressure drop induced by the liner. This effect is attributed to a modification of the flow induced by the acoustic wave. Thus, the flow is measured using PIV imaging technique and a comparison of velocity maps with and without sound excitation is performed. It is shown that the convection of large flow structures accompanies the sound amplification phenomenon. Some evidence of an hydrodynamic instability are thus given.

\section{Introduction}

Lined ducts are widely used to reduce noise emission, for example in turbofan engines. In the presence of flow, acoustic propagation is difficult to predict due to noise/flow interaction. This is especially true in the vicinity of the lined wall where the flow is highly vortical. Some analytical studies (based on linearized convected wave equation with Myers boundary conditions for the impedance) show the existence of many acoustic modes, and of two hydrodynamic modes in the presence of flow, one of which can be unstable [1]. Experimental demonstration of the existence of such instable modes has been made by Brandes and Ronneberger [2], who observed a large increase in acoustic transmission across a lined part of a cylindrical duct. More recently, an equivalent phenomenon was observed by Aurégan et al [3].

In the present paper, the flow above a lined wall of a flow duct is investigated when such an increase in acoustic transmission is present. The experimental setup is presented in section 2. The PIV experimental setup is presented in section 3. Information on the POD postprocessing is also given. Acoustic measurements showing a production of acoustic energy by the liner are then presented in section 4 together with mean pressure drop. Finally, results of the flow measurement above the liner are presented in section 5. Velocity fields are presented with or without acoustic excitation.

\section{Experimental Setup}

The experimental setup is presented in Fig 1. A rect-

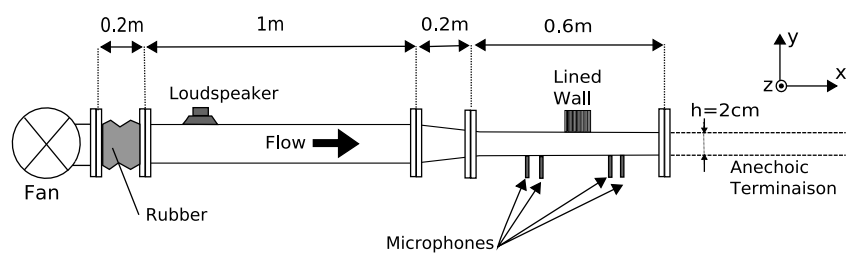

Figure 1: Top view of the flow rig. Scales are different in $x$ and $y$-directions.

angular pipe of cross section $8 \mathrm{~cm} \times 3 \mathrm{~cm}$ is fed by a fan. A loudspeaker is mounted on the pipe to introduce an acoustic wave. The cross section is then reduced via a $20 \mathrm{~cm}$ long contraction. Then comes the test section that has a rectangular cross section $8 \mathrm{~cm} \times 2 \mathrm{~cm}$ and is $60 \mathrm{~cm}$ long. It is made of aluminum but has some glass windows for optical access. One of the wall of the test section is acoustically treated with a liner. The test section and the lining material are shown in Fig. 2. The liner is equivalent to the one used in Ref [3] and comes from

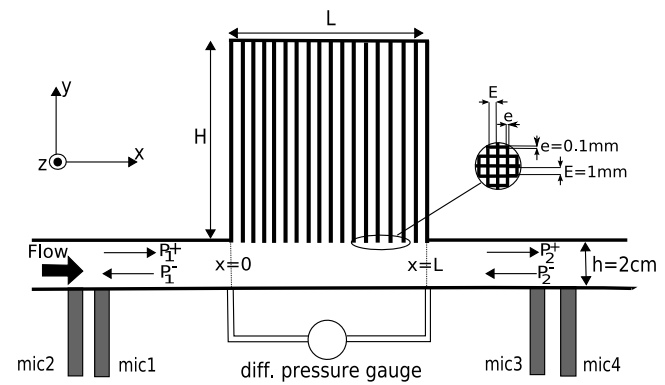

Figure 2: Top view of the test section. The cross section is rectangular, of height $h=2 \mathrm{~cm}$, and of width $8 \mathrm{~cm}$ along the $z$-direction.

automotive industry (catalytic converters). It consists of square ceramic resonators, side of which is $E=1 \mathrm{~mm}$. The resonators are separated by walls of thickness $e=0.1$ $\mathrm{mm}$. The length of the resonators is $H=8 \mathrm{~cm}$. This corresponds to a resonance frequency of $1 \mathrm{kHz}$. Hence, the acoustic transmission is expected to fall off at this frequency. The total length of the wall that is treated with the liner is $L=7.5 \mathrm{~cm}$. Finally the rig is ended by an anechoic terminaison.

An important parameter is the velocity of the flow. In the test section, the mean central velocity is up to 108 $\mathrm{ms}^{-1}$, which corresponds to a maximal Mach number $M=0.3$. Acoustic measurements (transmission) are performed for the plane mode using the four microphones shown in Fig. 2, using a swept sine excitation. A mean pressure drop can also be measured using a differential pressure gauge also shown in Fig. 2. The setup for the flow measurement is presented in the next section.

\section{$3 \quad$ PIV Setup and POD}

The flow is measured with a 2D Particle Image Velocimetry (2D PIV) technique [4]. The technique consists in taking two images of particles seeded in the flow. By correlating the two images one can deduce the displacement of the particles, and then the particle velocity by dividing the displacement by the time lag between the two images. An advantage of this technique is that it is not intrusive. It also makes it possible to obtain a instantaneous velocity map over some region of the flow. The measuring configuration for the present case in shown in Fig. 3. The laser sheet is introduced perpendicularly to the liner, and the camera takes images of particles in the PIV measurement area (see Fig. 3). This area is typically $2.5 \mathrm{~cm}$ long in the $x$-direction and $1.8 \mathrm{~cm}$ long in the $y$-direction. It does not cover the whole liner length, but the area can be moved along the liner. The measuring area can also be moved closer to 


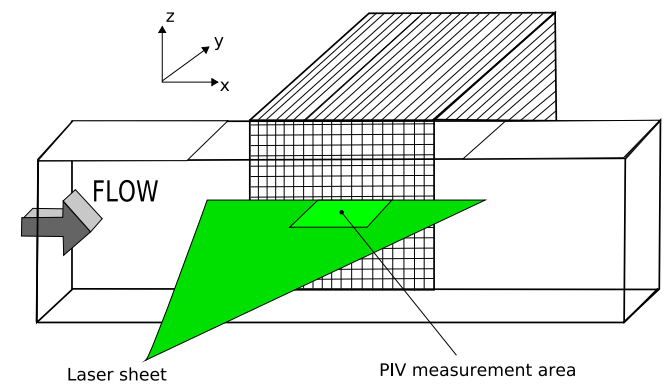

Figure 3: PIV Measurements Setup

the smooth wall facing the liner. The spatial resolution is $\Delta \sim 0.15 \mathrm{~mm}$. Note that spurious reflexions of the laser sheet on the walls means that the 3 to 4 first measured points close to the walls can contain large errors. 1000 velocity instantaneous fields are taken for each flow configuration at 1000 instants $t_{i}$. No synchronisation has been used when taking the images, and unfortunately the instants are given with a precision of $1 \mathrm{~ms}$, which is clearly not enough when compared to the frequency $1 \mathrm{kHz}$ encountered here. Hence, the time $t_{i}$ can be considered as unknown and completly random. We will use the following notations: $\mathbf{u}\left(x, y, t_{i}\right)$ is the instantaneous velocity vector with components $u$ and $v$ along $x$ and $y$ directions. The time averaged velocity vector is $\mathbf{U}(x, y)$ with components $U$ and $V$. Finally, the velocity fluctuation is $\mathbf{u}^{\prime}\left(x, y, t_{i}\right)=\mathbf{u}\left(x, y, t_{i}\right)-\mathbf{U}(x, y)$ with components $u^{\prime}$ and $v^{\prime}$. One can also consider the amplitude of the fluctuations given by the rms value of $\mathbf{u}^{\prime}$ that we will note $\mathbf{u}_{\mathbf{r m s}}$ with components $u_{r m s}$ and $v_{r m s}$. The $x$-axis origin is taken at the beginning of the treated portion of the duct, as shown in Fig. 2. The $y$-axis origin is taken at the position of maximal mean velocity, which is at the center of the channel. The lined wall is then at $y=0.01 \mathrm{~m}$ and the smooth wall is at $y=-0.01 \mathrm{~m}$. To compare the flow in the vicinity of the lined and smooth walls, a better vertical ordinate is the distance to the wall $w$, that will be used instead of $y$ when necessary.

To extract some useful information from the instantaneous velocity fields, use will be made of Proper Orthogonal Decomposition (POD). This decomposition allows to obtain a projection of the flow on particularly representative modes [5]. Contrary to a Fourier decomposition where modes are known in advance the POD provides "empirical" modes for the particular set of velocity fields under consideration. This POD is particularly useful when the flow is inhomogenous (ie when structures are present). When this is not so, the POD is equivalent to Fourier decomposition. The mean flow is substracted from the data before calculating the modes, thus the POD is performed on $\mathbf{u}^{\prime}$. The POD is:

$$
\mathbf{u}^{\prime}\left(\mathbf{x}, \mathbf{y}, \mathbf{t}_{\mathbf{i}}\right)=\sum_{\mathbf{j}=\mathbf{1}}^{\mathbf{N}_{\mathbf{t}}} \mathbf{a}_{\mathbf{j}}\left(\mathbf{t}_{\mathbf{i}}\right) \boldsymbol{\Psi}_{\mathbf{j}}(\mathbf{x}, \mathbf{y})
$$

where $N_{t}$ is the number of velocity fields. Here we will take $N_{t}=300$ fields out of the 1000 available. $t_{i}$ is the unknown time when the fields were taken. The real number $a_{j}$ are time coefficients without dimension. The modes $\boldsymbol{\Psi}_{j}(x, y)$ are vectors obtained by solving a Fredholm equation that will not be given here. Each mode
$\boldsymbol{\Psi}_{j}(x, y)$ is associated to its energy $\lambda_{j}$ also provided by the solution of the Fredholm equation. The modes are usually sorted in descending value of $\lambda$. Hence, the first mode is the most energetic.

The POD is particularly interesting when the dynamic of the flow can be synthesized using a few modes. In that case a reconstruction can be performed. For example, suppose the first two modes represent an important feature of the flow, a reconstructed field can be obtained through:

$$
\mathbf{u}_{\text {recons }}^{\prime}\left(x, y, t_{i}\right)=a_{1}\left(t_{i}\right) \Psi_{1}(x, y)+a_{2}\left(t_{i}\right) \Psi_{2}(x, y)
$$

Examples of PIV measurements together with POD postprocessing will be given in section 5 .

\section{Pressure/acoustic measurements}

On each side of the treated section, the acoustic wave is decomposed into complex incident and reflected components, that is $P_{1}^{+}$and $P_{1}^{-}$upstream of the liner, and $P_{2}^{+}$ and $P_{2}^{-}$downstream of the liner. A two microphones measurement on each side of the liner gives access to these quantities. One can thus calculate the acoustic transmission $T=P_{2}^{+} / P_{1}^{+}$, and the two reflexion coefficients $R_{1}=P_{1}^{-} / P_{1}^{+}$and $R_{2}=P_{2}^{-} / P_{2}^{+}$. The acoustic transmission $|T|$ is given as a function of frequency in Fig. 4 for several values of the Mach number $M$. With

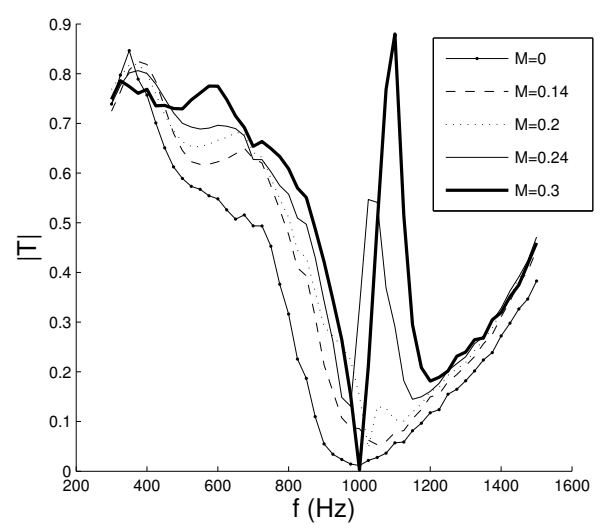

Figure 4: Acoustic transmission $|T|$.

no flow $(M=0)$ the transmission is close to 0 for the resonance frequency of the liner (around $1 \mathrm{kHz}$ ), as expected. When the Mach number increases, a hump is appearing in the transmission for a frequency that increases with the Mach number but keeps close to the resonance frequency of the liner. At $M=0.3$, the transmission reaches 0.9 at $1.1 \mathrm{kHz}$. The later value of the frequency corresponds to an increase of the temperature from $293 \mathrm{~K}$ to 306K at this Mach number. This hump in the transmission is unexpected, and a similar phenomenom has been observed previously in Refs [2, 3]. In Ref [3], the transmission can be to 3 , for slightly different conditions.

Another quantity of interest is the acoustic energy flux entering the liner, $E_{i n}$, given by

$$
\begin{aligned}
E_{i n} & =(1+M)^{2}\left|P_{1}^{+}\right|^{2}+(1-M)^{2}\left|P_{2}^{-}\right|^{2} \\
& =(1+M)^{2}\left|P_{1}^{+}\right|^{2}+(1-M)^{2}\left|R_{2} T\right|^{2}\left|P_{1}^{+}\right|^{2}
\end{aligned}
$$


The acoustic energy flux leaving the material is:

$$
\begin{aligned}
E_{\text {out }} & =(1+M)^{2}\left|P_{2}^{+}\right|^{2}+(1-M)^{2}\left|P_{1}^{-}\right|^{2} \\
& =(1+M)^{2}|T|^{2}\left|P_{1}^{+}\right|^{2}+(1-M)^{2}\left|R_{1}\right|^{2}\left|P_{1}^{+}\right|^{2}
\end{aligned}
$$

Thus a normalized acoustic energy dissipation can be calculated using the difference between these two fluxes, and is given in Fig. 5. A striking feature appears at

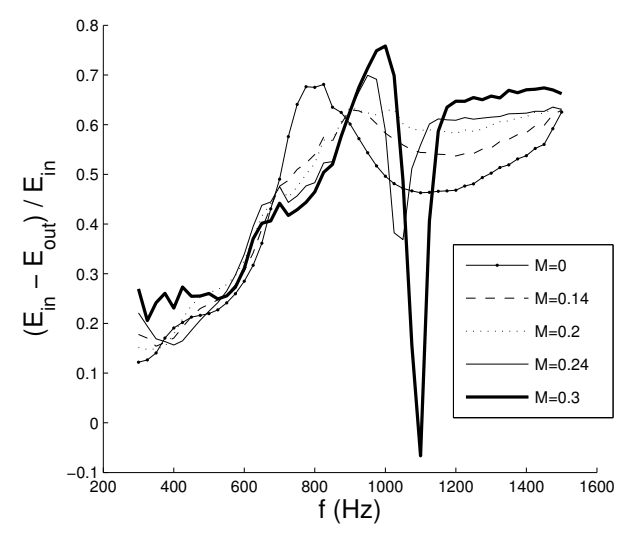

Figure 5: Acoustic energy dissipation by the liner.

$M=0.3$. The energy dissipation become negative at the same frequency $1.1 \mathrm{kHz}$ for which there was a hump in the acoustic transmission. This means that acoustic energy is indeed produced by the liner, and this is the opposite of what is expected from the liner.

To complete these acoustic measurements, the mean pressure drop across the liner has been measured at $M=0.3$ with acoustic excitation at a fixed incident level of $130 \mathrm{~dB}$, as well as without acoustic excitation. It is given in Fig. 6. It is seen that an increase in the

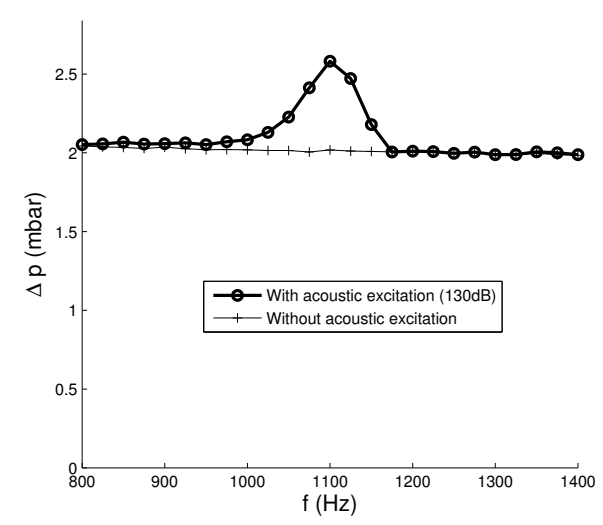

Figure 6: Mean pressure drop across the liner with or without acoustic excitation at $M=0.3$.

mean pressure drop of about $25 \%$ is seen at $1.1 \mathrm{kHz}$ when acoustic excitation is used. This shows that at this frequency, a large part of the flow above the liner is modified by the acoustic excitation.

\section{Flow measurement (PIV)}

To give an explanation to the large increase in acoustic transmission observed above, the flow above the liner is now investigated using $2 \mathrm{D}$ PIV as presented in section 3. Of special interest is the comparison between the flow obtained with ('Ac') and without ('NoAc') acoustic excitation, and between the flow close to the lined wall and that close to the smooth (rigid) wall at a fixed axial position $x$. In any case, the Mach number is fixed at $M=0.3$, and the optional acoustic excitation has a frequency of $1.1 \mathrm{kHz}$, corresponding to that for which an acoustic phenomenom has been observed in the previous section.

First let us consider the velocity statistics. As an example the profile of the rms value of the axial velocity, $u_{r m s}$, is given for several cases in Fig. 7, at two different axial positions: $x=0.002 \mathrm{~m}$ (beginning of the treated part of the duct), and $x=0.07 \mathrm{~m}$ (the end of the treated part of the duct). At $x=0.002 \mathrm{~m}$, we see no difference

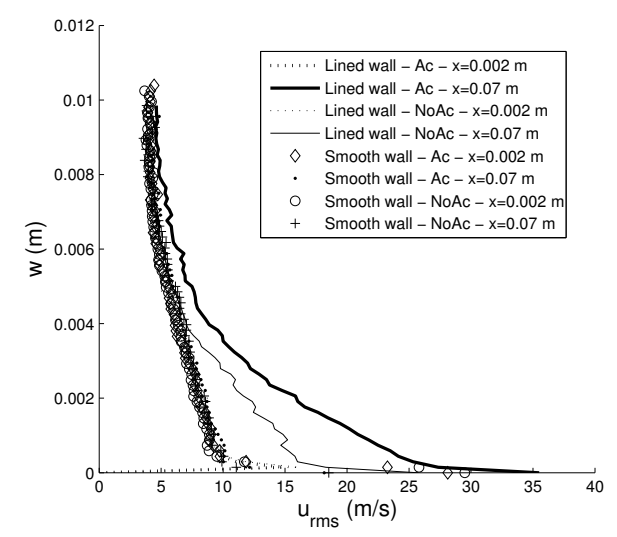

Figure 7: Profiles of the axial velocity fluctuations, $u_{r m s} . w$ is the distance to the wall.

between the smooth and lined walls, with or without excitation. Similar observations are done upon $U, V$, and $v_{r m s}$ (not shown). This means that at the entry of the treated region, no phenomena has appeared yet, whatever the case. The profiles at $x=0.07 \mathrm{~m}$ for the smooth wall are close to those at $x=0.002 \mathrm{~m}$. It means that the flow in the vicinity of the rigid wall at the end of the treated region has not changed compared to the flow at the entry of the region, even with acoustic excitation present. In Fig. 7, only two profiles actually stand out, these are the fluctuation profiles for the lined wall at $x=0.07 \mathrm{~m}$, at the exit of the treated region in the vicinity of the lined wall. These fluctuations at the exit are much bigger than at the entry. This is true with and without excitation, yet the fluctuations are much larger with excitation. This shows that the acoustic excitation has a deep impact on the flow fluctuations above the liner.

In the following, we try to establish a relation between instantaneaous features of the flow and the previous observations on first-order statistics. An example of instantaneous axial velocity map is given in Fig. 8 . The field is in the vicinity of the upper (lined) wall and at the end of the treated section $(0.045 \mathrm{~m}<x<0.07 \mathrm{~m})$. It is particularly clear that a structure is present along the lined wall. Such a large structure is not visible on the bottom (rigid) wall. 


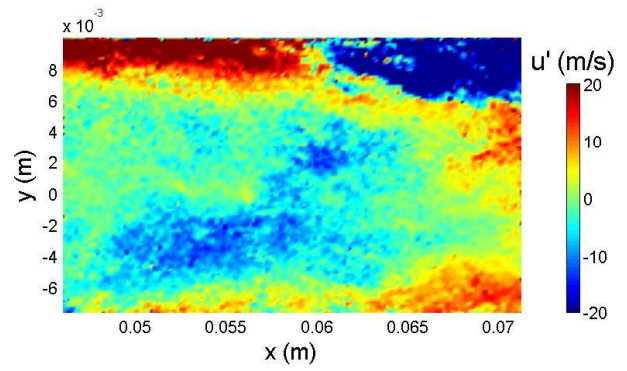

Figure 8: Example of instantaneouas axial velocity $u^{\prime}$.

With acoustic excitation at $1.1 \mathrm{kHz} . \quad M=0.3$.

The presence of a structure makes a POD investigation interesting. The first two modes of the POD decomposition for the case with acoustic excitation are shown in Figs. 9 and 10. These two modes gather 50\% of the fluctuating energy. Both modes show clearly

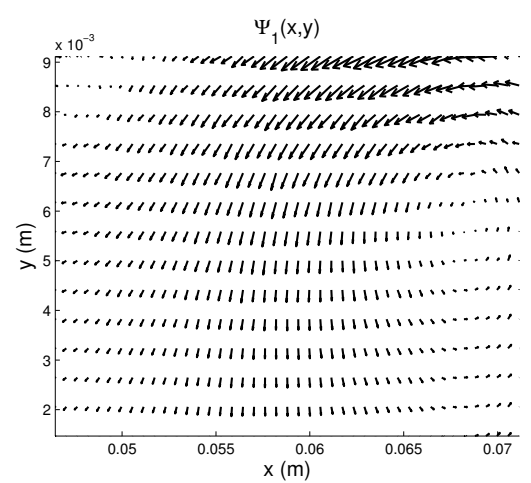

Figure 9: First POD mode.

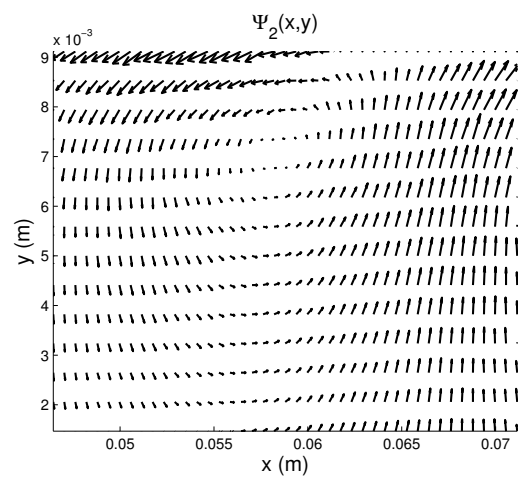

Figure 10: Second POD mode.

a vortex with an axial offset between the two modes. This means that the vortex is probably convecting. The time coefficients in Eq. 1 vary quite arbitrarily since no phase locking was used. Nevertheless, information can be gained by plotting a phase diagram of $a_{2}$ versus $a_{1}$, which is done in Fig. 11. In this figure, a velocity field at time $t_{i}$ is represented by a dot, and we have $N_{t}=300$ dots. It is observed that the dots describe rather well a circle shape. Hence we can write $a_{1}=R \cos (\phi)$ and $a_{2}=R \sin (\phi)$, where $\phi$ is a phase angle. This phase angle can be used to sort the $N_{t}$ velocity fields by ascending value of $\phi$ or to make phase averages. To do this we first define ten phase $\phi_{k}, \mathrm{k}=1 \ldots 10$, corresponding to the ten slices shown in Fig. 11. They are given by:

$$
\phi(k)=(k-0.5) \frac{2 \pi}{10} \quad k=1 \ldots 10
$$

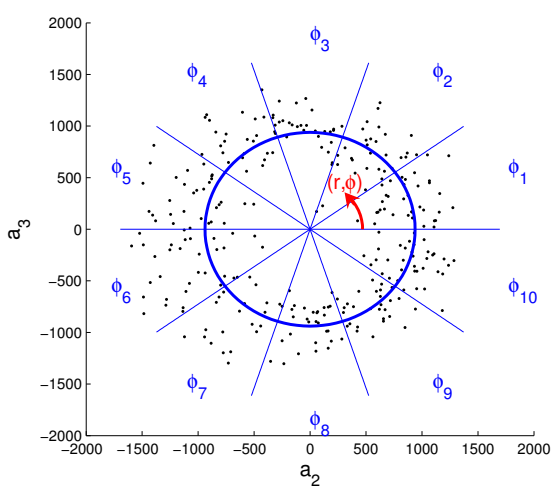

Figure 11: Phase diagram $a_{2} / a_{1}$.

Second, we can average all the velocity fields falling in a particular slice. Thus we define a phase-averaged fluctuating velocity field by:

$$
\mathbf{u}_{\text {phase }}^{\prime}(k)=\sum_{i ;(k-1) \frac{2 \pi}{10} \leq \phi(i)<k \frac{2 \pi}{10}} \mathbf{u}^{\prime}(i) \quad k=1 \ldots 10
$$

We can do the same for any quantity that depends only on the instant $i\left(\in\left[\begin{array}{ll}1 & N_{t}\end{array}\right]\right)$ of the velocity field. A phase averaged time coefficient for mode 1 is given by

$$
a_{1, p h a s e}(k)=\sum_{i,(k-1) \frac{2 \pi}{10} \leq \phi(i)<k \frac{2 \pi}{10}} a_{1}(i) \quad k=1 \ldots 10
$$

A phase averaged time coefficient for mode $2, a_{2, \text { phase }}$, is defined similarly. These two coefficients are plotted versus the phase in Fig. 12. The curves describing these phase-averaged coefficients are offseted sine curves, which is nothing but another way of representing the circle in Fig. 11. Hence, although no synchroni-

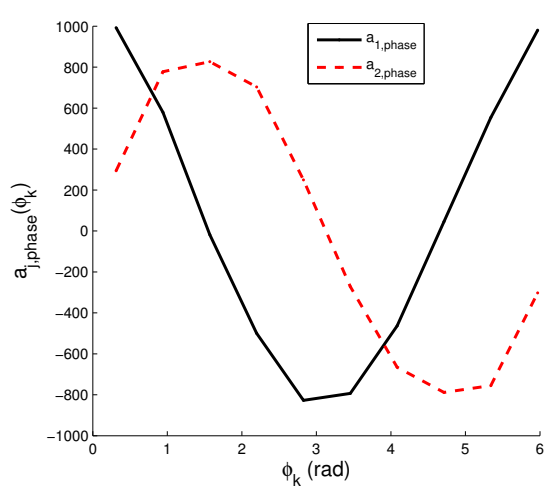

Figure 12: Evolution of the phase averaged time coefficients with the phase.

sation was used for the measurement, we are able using the previous method to recover a periodicity in the velocity fields. To summarize, we have defined 10 phase angles $\phi_{k}$ and ten phase-averaged fluctuating velocity fields. We have also 10 coefficients $a_{1, \text { phase }}$ and $a_{2, \text { phase }}$ for the modes $\boldsymbol{\Psi}_{\mathbf{1}}$ and $\boldsymbol{\Psi}_{\mathbf{2}}$. This in particular allows us to reconstruct a field with two modes. Eq. 2 has to be replaced by:

$$
\begin{aligned}
\mathbf{u}_{\text {recons,phase }}^{\prime} & (x, y, k)=a_{1, \text { phase }}(k) \boldsymbol{\Psi}_{\mathbf{1}}(x, y) \\
& +a_{2, \text { phase }}(k) \boldsymbol{\Psi}_{\mathbf{2}}(x, y) \quad k=1 \ldots 10
\end{aligned}
$$


This is a phase-reconstructed fluctuating velocity using the first two modes only, and is avaible for each of the ten phases considered.

Figs. 13 and 14 show respectively the phase-averaged and phase-reconstructed velocity vector field, both at phase $\phi_{1}$. We see that these fields are quite simi-

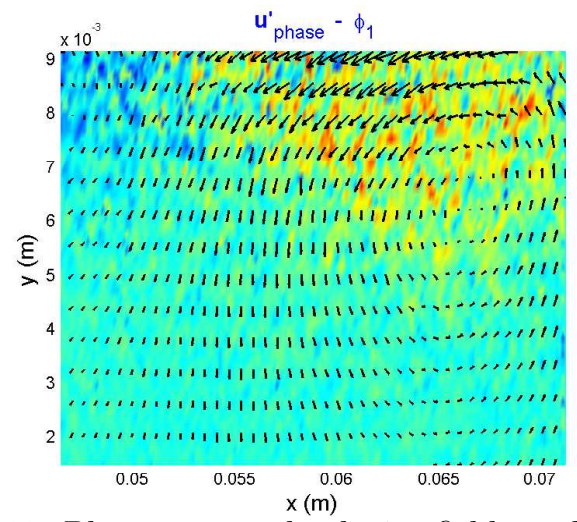

Figure 13: Phase averaged velocity field at phase $\phi_{1}$. The instantaneous velocity fields falling in slice $\phi_{1}$ of

Fig. 11 have been averaged to obtain this field.

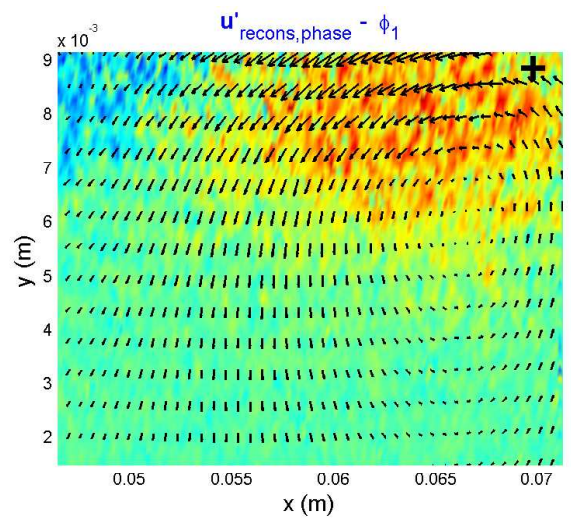

Figure 14: Phase averaged reconstructed velocity field at phase $\phi_{1}$ according to Eq. 10.

lar (the reconstructed field is much smoother) and represent a vortex. At the other phases $\phi_{2} \ldots \phi_{10}$ (not shown) the velocity field represent a vortex at other positions. Hence, a major future of the velocity fluctuation is a vortex convected along the liner. A spatial Fourier transform show that the associated axial wavelength is about $\lambda \sim 0.04 \mathrm{~m}$. A full period corresponds to 2 conterrotating vortices and only about one half of this wavelength is visible in Figs 13-14. Based on the wavelength and the frequency of $1.1 \mathrm{kHz}$, the convection speed of the vortex is $u_{\text {conv }}=\lambda f=44 \mathrm{~ms}^{-1}$. It is interesting to plot the evolution of the velocity at a fixed point of the domain. This point is taken to be at $x=0.07 \mathrm{~m}$ and $w=0.0012 \mathrm{~m}$, and is shown by a black cross in Fig. 14 . Both the phase-averaged and reconstructed fluctuating axial velocity are given in Fig. 15 as a function of the phase. For more clarity, the fields have been duplicated, and the phase goes up to $6 \pi$. The phase-averaged velocity has a sine shape. The reconstructed velocity is very close to it, which shows that the first two POD modes are sufficient to describe the phase-averaged dynamics of the flow. A striking fact in Fig. 15 is the magnitude of the phase-averaged velocity fluctuation, that reaches

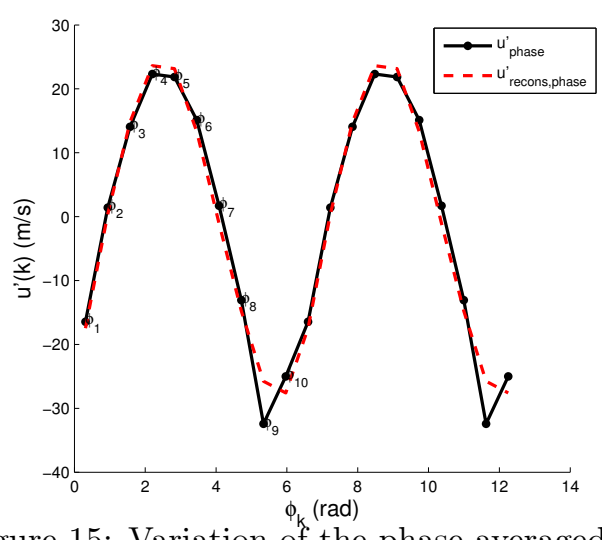

Figure 15: Variation of the phase-averaged and reconstructed axial velocity at the point located at $x=0.07 \mathrm{~m}, w=0.0012 \mathrm{~m}$.

more than $20 \mathrm{~ms}^{-1}$. The peak velocity for the transverse velocity (not shown) is about $10 \mathrm{~ms}^{-1}$. Thus the effect of the acoustic excitation on the flow field is seen to be quite important.

\section{Conclusion}

At sufficiently high Mach numbers, a liner consisting of quarter-wavelength resonators can amplify an incident sound wave at its resonance frequency. This acoustic behaviour has been related to the flow field above the liner. A phase-averaged velocity shows convected vortex along the liner, with a periodic velocity at a fixed point. This supports the hypothesis of an instable hydrodynamic mode.

\section{Acknowledgments}

The support from FRAE under IROQUA/COMATEC research programme is gratefully acknowledged.

\section{References}

[1] S. W. Rienstra, "A classification of duct modes based on surface waves", Wave motion 37, 119135 (2003)

[2] M. Brandes, D. Ronneberger, "Sound Amplification in flow ducts lined with a periodic sequence of resonators", 1th AIAA/CEAS Aeroacoustics Conference, AIAA paper 95-126 (1995)

[3] Y. Aurégan, M. Leroux, "Experimental evidence of an instability along an impedance wall with flow", accepted for publication in J. sound Vib. (2008)

[4] R. J. Adrian, "Particle Imaging technique for experimental fluid mechanics", Ann. Rev. Fluid Mech. 23, 261-304 (1991)

[5] G. Berkooz, P. Holmes, and J. L. Lumley, "The Proper Orthogonal Decomposition in the analysis of turbulent flows" Annual Review of Fluid Mechanics 25, 539-575 (1993) 